\title{
A collaborative approach to exercise provision for people with Parkinson's - a feasibility and acceptability study of the
} PDConnect programme [version 1; peer review: 2 approved with reservations]

\author{
Julie Jones (iD)1, Lyndsay Alexander (iD)1, Elizabeth Hancock¹, Kay Cooper (iD)1,2 \\ ${ }^{1}$ School of Health Sciences, Robert Gordon University, Aberdeen, AB10 7QT, UK \\ ${ }^{2}$ AHP Directorate, NHS Grampian, Aberdeen, UK
}

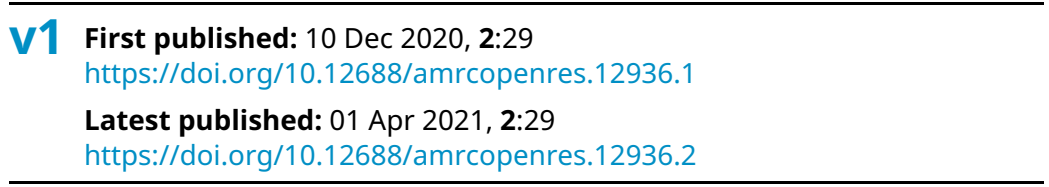

\section{Abstract}

Background: Exercise has been shown to be beneficial for people with Parkinson's (PwP), limiting the rate of decline of motor and nonmotor symptoms, with emerging evidence associating exercise with a neuroprotective effect. Current exercise provision is time-limited, and delivered in the absence of strategies to support long-term adherence to exercise. With a growing Parkinson's population, there is a need to develop long-term sustainable approaches to exercise delivery. The primary aim of this study is to assess the feasibility and acceptability of a multicomponent intervention (PDConnect) aimed at promoting physical activity, and self-management for PwP.

Methods: A convergent fixed parallel mixed methods design study will be undertaken. The study aims to recruit 30 PwP, who will be randomly allocated into two groups: (i) the usual care group will receive physiotherapy once a week for six weeks delivered via Microsoft Teams. (ii) The PDConnect group will receive physiotherapy once a week for six weeks which combines exercise, education and behaviour change interventions delivered by NHS Parkinson's specialist physiotherapists via Microsoft Teams. This will be followed by 12 weekly sessions of group exercise delivered on Microsoft Teams by fitness instructors specially trained in Parkinson's. Participants will be then contacted by the fitness instructors once per month for three months by video conferencing to support exercise engagement. Primary feasibility data will be collected during the study, with acceptability assessed via semi-structured interviews at the end. Secondary outcomes encompassing motor, non-motor and health and well-being measures will be assessed at baseline, at six, 18, and 30 weeks.

Discussion: This pilot study will establish whether PDConnect is feasible and acceptable to PwP. This will provide a platform for a

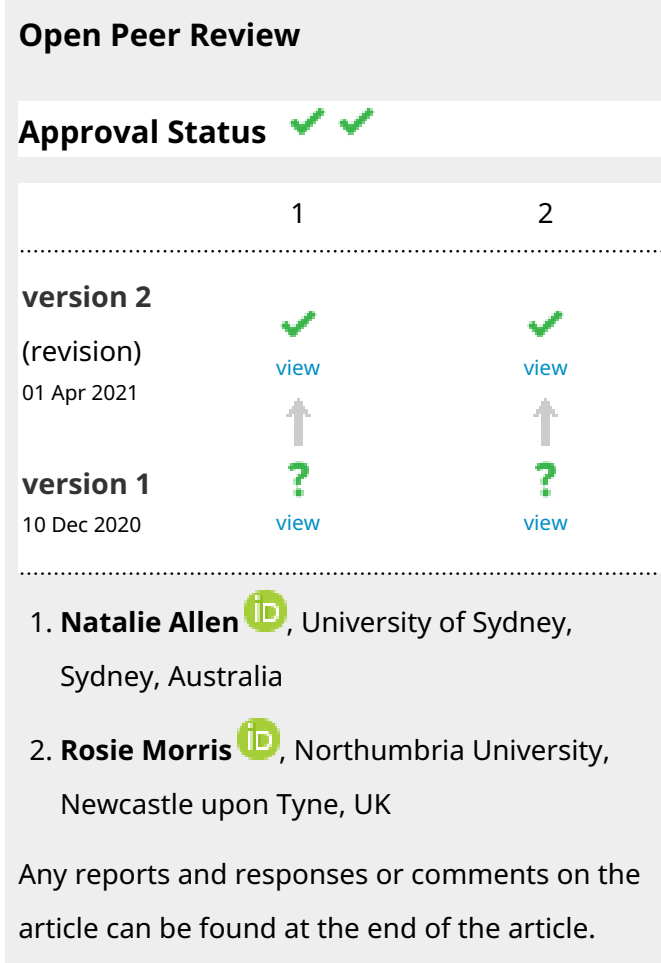


larger evaluation to assess the effectiveness of PDConnect at increasing exercise participation and self-management within the Parkinson's Community.

Trial registration: Registered on ISRCTN (ISRCTN11672329, $4^{\text {th }}$ June 2020).

Keywords

Parkinson's, exercise, behaviour change, empowerment, partnership

\begin{tabular}{|c|c|}
\hline 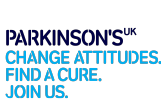 & $\begin{array}{l}\text { This article is included in the Parkinson's } U \\
\text { gateway. }\end{array}$ \\
\hline
\end{tabular}

Corresponding author: Julie Jones (j.c.jones@rgu.ac.uk)

Author roles: Jones J: Conceptualization, Funding Acquisition, Methodology, Project Administration, Resources, Writing - Original Draft Preparation, Writing - Review \& Editing; Alexander L: Funding Acquisition, Methodology, Supervision, Writing - Review \& Editing; Hancock E: Funding Acquisition, Methodology, Supervision, Writing - Review \& Editing; Cooper K: Funding Acquisition, Methodology, Supervision, Writing - Review \& Editing

Competing interests: No competing interests were disclosed.

Grant information: This study is jointly funded by the Chief Scientist Office and Parkinson's UK. Grant award number: F1901. The Clinical Academic Fellowship was awarded to the principal researcher Julie Jones.

The funders had no role in study design, data collection and analysis, decision to publish, or preparation of the manuscript.

Copyright: @ 2020 Jones J et al. This is an open access article distributed under the terms of the Creative Commons Attribution License, which permits unrestricted use, distribution, and reproduction in any medium, provided the original work is properly cited.

How to cite this article: Jones J, Alexander L, Hancock E and Cooper K. A collaborative approach to exercise provision for people with Parkinson's - a feasibility and acceptability study of the PDConnect programme [version 1; peer review: 2 approved with reservations] AMRC Open Research 2020, 2:29 https://doi.org/10.12688/amrcopenres.12936.1

First published: 10 Dec 2020, 2:29 https://doi.org/10.12688/amrcopenres.12936.1 


\section{Introduction}

Parkinson's is the second most common neurodegenerative condition $^{1}$, with global prevalence estimated to exceed 12 million by $2040^{2}$. Parkinson's has over 40 recognised motor and non-motor symptoms, which negatively affect function and quality of life $(\mathrm{QoL})^{3}$. Despite major advances in the understanding of Parkinson's, the cure remains elusive, as do effective long-term interventions. Medical management focusses on limiting the impact of symptoms, but medication has a time-limited effect and is associated with substantial side effects. This has fuelled the need to look at alternative strategies for those living with Parkinson's. Exercise has been hailed as the new medicine for Parkinson's and is no longer viewed as a complementary intervention, but is considered of equal importance to medication and as such needs to be undertaken daily ${ }^{3}$. Exercise is defined as activities, which are planned, structured, and purposeful, with the intention of improving and/or maintaining one or more components of physical fitness ${ }^{4}$. Exercise is a subcategory of physical activity, the latter being an umbrella term which encompasses bodily movements produced by skeletal muscles. Physical activity includes a wide range of behaviours including gardening, housework and leisure related activities ${ }^{4}$.

The progressive nature of Parkinson's ${ }^{5}$ limits participation in exercise and initiates a vicious cycle of inactivity. Inactivity results in deconditioning, leading to reductions in muscle strength, flexibility, balance, aerobic fitness and an increased risk of falls ${ }^{6}$. This is compounded further by non-motor symptoms such as fatigue and depression, which reduce motivation to be active ${ }^{7}$. These factors culminate in reduced socialisation, and subsequently QoL ${ }^{3,8,9}$. The long-term sequela being that the body becomes deconditioned not only as a result of Parkinson's, but also due to inactivity, as illustrated in Figure 1. Consequently, up to $80 \%$ of $\mathrm{PwP}$ are classed as sedentary ${ }^{10}$, with exercise levels declining from diagnosis ${ }^{11}$. The impact of Covid-19 has further impacted on physical activity levels ${ }^{12}$. Consequently, PwP have reported deterioration in Parkinsonian symptoms, and mental health and well-being due to restrictions put in place due to Covid-19 ${ }^{13}$. Regular participation in exercise has been shown to slow down the rate of symptom progression ${ }^{14-17}$ in Parkinson's; therefore, there is an urgent need for health interventions, which support PwP to adopt long-term exercise habits.

Exercise participation among $\mathrm{PwP}$ is associated with improvements in strength, balance, gait, and cognition ${ }^{6,18,19}$ with emerging evidence linking high intensity exercise with a potential disease-modifying effect ${ }^{17,20-22}$. Owing to the diversity of parkinsonian symptoms, sole reliance on any one form of exercise is not advocated; instead a multimodal approach is required $^{23}$. Research suggests that frequency and intensity of exercise are key determinants of successful exercise prescription, with guidelines advocating moderate to high intensity exercise conducted five times a week ${ }^{24,25}$. Moreover, exercise needs to be prescribed for a minimum of ten weeks, to promote the physiological adaptation and skill acquisition required to develop self-confidence with exercise. However, the current healthcare system does not have capacity to support high intensity input $^{26-28}$, nor support exercise long-term, and therefore alternative models to delivery of exercise for PwP are urgently required ${ }^{29}$.

Simply instructing PwP to exercise is not effective ${ }^{23}$. Rather, exercise needs to be delivered as a package of care, which can be delivered long term to support the development of a daily exercise habit. Healthcare policy proposes that self-management programmes are the mechanism to effectively manage long-term conditions ${ }^{26}$ and support sustained behaviour change. Self-management evolved from the Chronic Care Model proposed by Wagner et al. $(1998)^{30}$, emphasising the importance of organisational change to promote practical, supportive, evidencebased interactions between an informed patient and a proactive practice team. Self-management programmes aim to empower participants through the development of self-efficacy. However, PwP report that access to exercise and ability to self-manage are their greatest unmet needs ${ }^{31}$. Combining exercise with behaviour change and education, as a combined health intervention, has been proposed to be more effective than any one component delivered in isolation ${ }^{32}$. Therefore, delivering exercise as part of a self-management programme embedded with behaviour

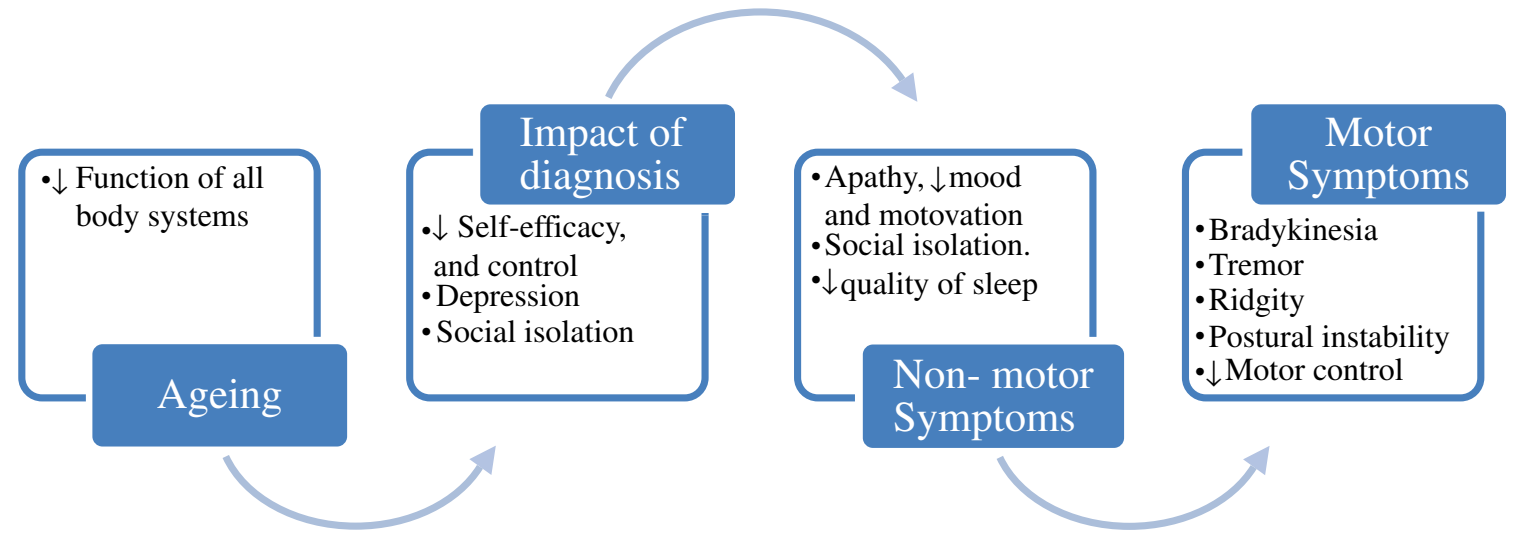

Figure 1. Complex interplay of factors which negatively impact physical activity participation among people with Parkinson's. 
change strategies could assist the development of exercise self-efficacy, self-actualisation and empowerment of PwP to assume a partnership role within their care.

The emergence of the Covid-19 pandemic has significantly impacted on the capacity for face-to-face interventions, and thus there is an urgent need to develop alternative solutions to patient engagement, and delivery of treatment, in particular exercise. The role of telehealth, using online means to deliver exercise, may be a practical solution to the evolving challenges of delivering health during the Covid-19 pandemic and beyond, with digital health being central to Scottish government healthcare delivery plans $^{33}$. The potential value of telemedicine as a mechanism to support monitoring of symptoms, and medication management is widely acknowledged ${ }^{34-36}$. PwP have reported high satisfaction with online support ${ }^{37}$, with some preferring online delivery compared with face-to-face ${ }^{38}$. Online delivery offers a flexible means to delivering exercise which can be delivered within the home, removing environmental barriers and mitigating interpersonal barriers, providing exercise in a de-medicalised environment, reducing participant anxiety and costs for providers. Home-based exercise has been shown to be effective ${ }^{39}$, feasible, and safe even when delivered at moderate to high intensity for $\mathrm{PwP}^{40}$. The use of a video guided exercise health app has been shown to be feasible among $\mathrm{PwP}^{41}$, although adherence to the programme was poor, although this was self-guided programme, which may explain why few participants met their activity goals and the high dropout following baseline measures. Suggesting that supervision is needed to promote and maintain engagement in exercise. However, the feasibility and acceptability of remotely delivered online exercise for PwP has yet to be fully explored.

Following Medical Research Council Guidelines for Designing Complex Interventions ${ }^{42}$, this study aims to explore the feasibility and acceptability of an online collaborative approach to exercise, delivered by specially trained NHS physiotherapists and fitness instructors, which incorporates individualised exercise, education, and behaviour change techniques to support long-term exercise participation for PwP.

\section{Protocol}

Protocol Version: Version 3, 13 ${ }^{\text {th }}$ November 2020

\section{Objectives}

To determine the feasibility and acceptability of a remotely delivered multi-component intervention (PDConnect) aimed at promoting individualised exercise, physical activity and self-management among community dwelling adults with Parkinson's.

\section{Study design}

A fixed parallel convergent mixed methods design will be employed.

\section{Setting}

This study will be conducted in North East Scotland delivered exclusively online using Microsoft Teams.

\section{Participants}

This study aims to recruit $30 \mathrm{PwP}$, which aligns with prior feasibility studies ${ }^{43,44}$ and will allow sufficient numbers for group exercise. Using convenience sampling, recruitment will be carried out in partnership with NHS consultant neurologists and geriatricians. The consultants will identify eligible PwP who meet the study inclusion criteria (Table 1 ) and will provide them with a participation information sheet (Extended data ${ }^{45}$ ) and the principal researcher's contact details. Those contacting the researcher will be given opportunity to ask any further questions and will be screened to ensure safety to exercise. PwP who are considered eligible will be offered an appointment within two weeks to undertake consent and baseline measures within their own home, prior to randomisation to either PDConnect or usual care (Figure 2).

\section{Table 1. Study inclusion criteria.}

\begin{tabular}{|c|c|}
\hline Inclusion criteria & Exclusion criteria \\
\hline $\begin{array}{l}\text { - } \text { Confirmed diagnosis of Parkinson's } \\
\text { - } \text { Stage I-III Hoehn and Yahr Scale } \\
\text { score of } \leq 2 \text { on the Unified Parkinson's } \\
\text { disease Rating Scale (UPDRS) item } 29 \\
\text { - } \text { Able to walk independently with or } \\
\text { without a walking aid further than 100m } \\
\text { - Stable medication for more than three } \\
\text { weeks } \\
\text { - Able to speak and understand English } \\
\text { without assistance } \\
\text { Own or have access to a laptop or tablet } \\
\text { with a webcam, which is compatible with } \\
\text { Microsoft Teams. }\end{array}$ & $\begin{array}{l}\text { - Secondary or atypical Parkinsonism } \\
\text { - Severe, unpredictable episodes of motor } \\
\text { fluctuation } \\
\text { - Use of medications known to interfere with } \\
\text { cognitive function } \\
\text { - History of neurological diseases other than } \\
\text { Parkinson's } \\
\text { - Any unstable mental or physical condition } \\
\text { that prevent consenting and participating } \\
\text { in exercise. } \\
\text { - Unstable or uncontrolled medical } \\
\text { conditions }\end{array}$ \\
\hline
\end{tabular}




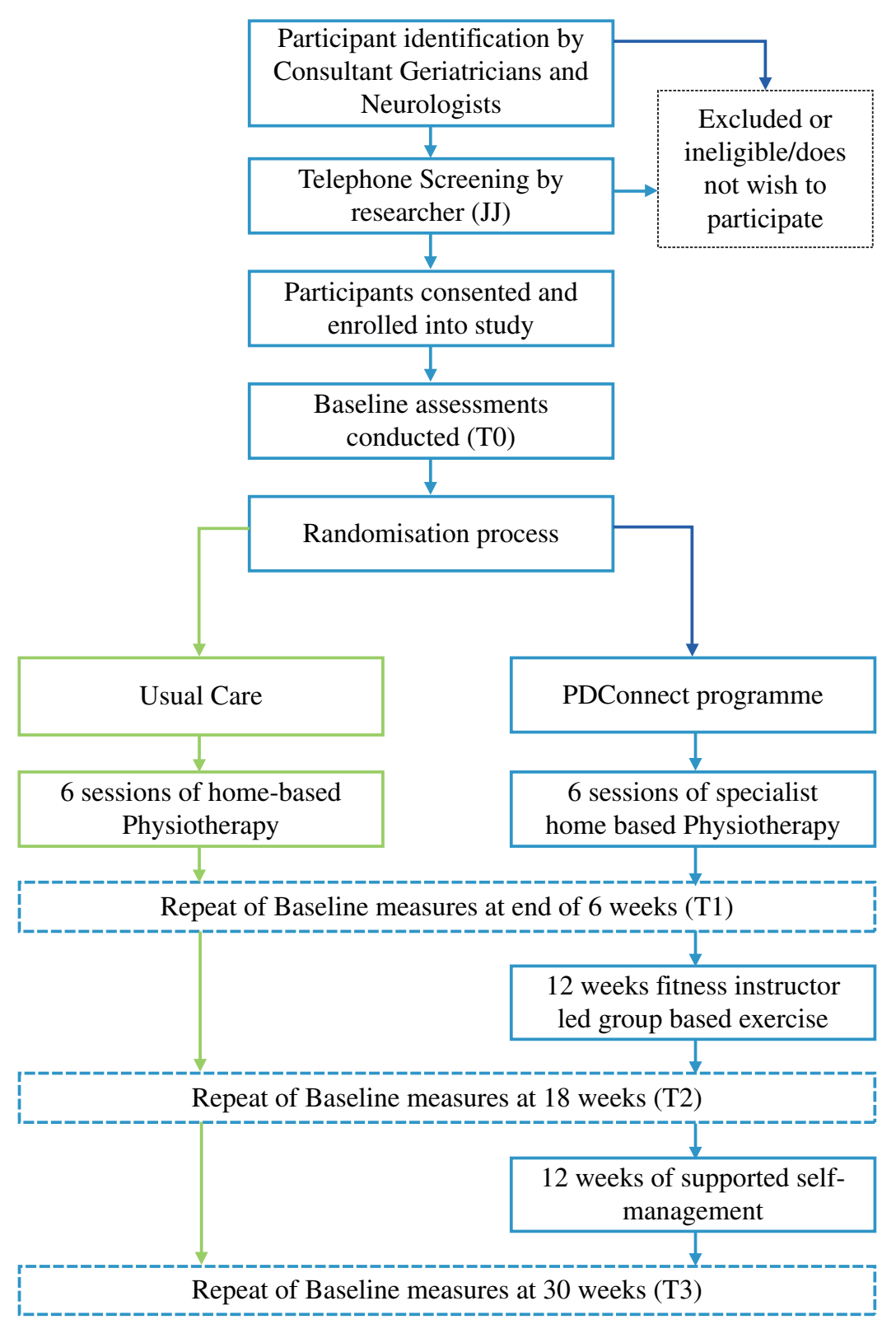

Figure 2. Study flowchart.

\section{Randomisation and blinding}

Randomisation will be conducted by a Chartered Statistician independent to the study. Stratified random sampling by disease stage (Hoehn and Yarh stage), age, and gender will be employed to ensure balance at baseline. Using computer-generated random number sequencing (Excel, Microsoft Corporation) in a ratio of $1: 1$, a random number will be placed in a sealed, sequentially numbered, opaque envelope. PwP will be allocated randomly by extracting the random number from the envelope and will be notified of group allocation via letter. Group allocation will be concealed from the principal researcher until the end of the study, so that they remain blind to group allocation. Owing to the nature of the intervention being exercise, blinding of PwP is not possible. General Practitioners and Parkinson's Consultants will be informed of their patients' involvement within the study.

\section{Physiotherapist/fitness instructors}

Four NHS Physiotherapists will be recruited via purposeful sampling. Eligible Physiotherapists will be Agenda for Change Band 6 or above, with a minimum of two-years clinical experience including neurology and geriatrics. The Physiotherapists will be randomly assigned to deliver either PDConnect $(n=2)$ or usual care $(n=2)$, by the use of sealed envelopes provided by the research assistant. Two Fitness 
Instructors will be recruited from the University Sport facility and will be Register of Exercise Professionals (REPS) accredited (or equivalent). The two Physiotherapists and Fitness Instructors delivering PDConnect will participate in a blended learning training package aimed at developing expertise in exercise prescription for PwP and behaviour change techniques. Those physiotherapists delivering usual care will not receive any training.

\section{Intervention}

Usual care. Usual care is defined as physiotherapy delivered by community-based physiotherapists (Band 6 or above) who have not received specialist postgraduate training in treatment and management of Parkinson's. PwP will receive six one-to-one physiotherapy sessions lasting up to an hour delivered online via Microsoft Teams. Each session will include assessment, treatment, goal setting and intervention delivery, and will be supplemented by a home exercise programme (HEP). Treatment choices will be guided by participant need, as per standard practice.

PDConnect. PDConnect is an evidence-informed exercise intervention underpinned by empowerment theory, with the aim of providing PwP with a toolkit of behaviour change techniques (BCTs) to promote participation in exercise. The PDConnect programme combines specialist physiotherapy, group-based exercise and self-management, with education and behaviour changes strategies threaded throughout. The programme consists of three components: i) six sessions of one-to-one specialist physiotherapy delivered at home; ii) 12 weekly sessions of group-based exercise; iii) 12 weeks of self-management, where participants will be contacted monthly. Each component will be delivered online using Microsoft Teams.

Specialist physiotherapy: Participants will receive six one-to-one physiotherapy sessions over six weeks, to promote continuity of treatment and develop a foundation to promote behaviour change and self-confidence with exercise engagement. Each one-hour session will encompass exercise, embedded with education and behaviour change interventions, and development of a HEP. Key BCTs will include: problem solving, identification of problems and developing self-awareness, and in the later stages self-belief, reward, and regulation. Exercise prescription will encompass strength, aerobic, balance, cognitive, gait, and functional components, with a focus on functional movement patterns, quality and amplitude of movement, in dual and single task activities as per the European physiotherapy guidelines ${ }^{46}$. Participants will be encouraged to work at a moderate to high intensity, defined as "somewhat hard" on the Borg Rating of Perceived Exertion scale ${ }^{47}$. Exercises will be mutually selected and delivered in an educational manner, to promote understanding, motivation and adherence.

Participants will be supported to develop a weekly exercise activity planner and will record all activity within an activity diary. One-to-one sessions will be supplemented by a HEP aligning with the participant's treatment goals and exercises will be selected from the REHABGuru (Potters Bar, UK) exercise library.
Participants will be encouraged complete their HEP five times a week, for a minimum of 30-minutes, and to record all exercise within their activity diary.

12-week group exercise: Acknowledging the value and support that family and carers can play in motivating PwP, family and carers will also be invited to attend, following basic health screening. Based on current research, the class will run for a minimum of 60-minutes, encompassing ten exercise stations, spending four minutes at each station ${ }^{4}$. Each station will have four levels of difficulty, tailored to individual ability. Informed by Parkinson's guidelines, exercises will include; mobility, strengthening, aerobic, balance, cognitive, goal-oriented components, with an emphasis on large amplitude movements and intensity of effort. All participants will receive an introduction to each exercise, encompassing key teaching points, practical demonstration, proposed benefits and purpose of the exercise. Videos of each exercise will be available on Microsoft Teams to prior to participating. Throughout the class, the fitness instructor will be present to provide feedback, refine technique and progress participants as appropriate. The remaining 30-minutes of the class is allocated to group-based discussion facilitated by the fitness instructor. The purpose of these discussions is to promote shared experience and learning.

12 weeks self-management: During this phase participants will exercise independently, following the HEP which has been developed during the programme. Participants will receive a 20-minute phone or video call every month from the fitness instructor, to review and adapt the HEP as required and to support problem solving to overcome any barriers that may have arisen.

\section{Outcome measurement}

Primary outcome measurement. A mixed methods approach will be adopted to establish feasibility, acceptability and fidelity. As such, data will be collected on feasibility and acceptability as outlined below:

Feasibility: Feasibility will be assessed by collecting data on the following:

- Recruitment and retention rates

- Adherence rates

- Time required to recruit to target

- Number of eligible participants required to recruit target sample size

- Number of PwP who complete each aspect of PDConnect

- Feasibility of testing procedures and data collection methods, including completion rates of outcome measures

Acceptability: Prospective acceptability (reasons for not taking part/discontinuation or dropping out) will be assessed via a survey. Semi-structured interviews will be conducted on completion of the study to explore the potential motivators and barriers associated with the PDConnect programme and experiences of those participating within the study (i.e. 
physiotherapists, fitness instructors and $\mathrm{PwP}$ ). Interviews will be conducted by the principal researcher, using Microsoft Teams video conferencing. Interviews involving those delivering the intervention will be conducted using Microsoft Teams. Interviews will be conducted following the approached outlined by Creswell and Plano-Clark $(2011)^{48}$, adopting a topic guide (Extended data $^{45}$ ) to inform conduct of the interviews. Interviews will be recorded via digital voice recorder and fully transcribed after the event. All transcripts will be sent to interviewees following the interview, to confirm accuracy.

Fidelity assessment: A random sample of six physiotherapy interventions and three group exercise sessions will be videoed to facilitate fidelity assessment of intervention delivery, which will be conducted by the researcher.

Secondary outcome measurement. Although this study is not powered to detect statistically significant changes in clinical outcome measures, secondary outcomes (measured at baseline, at six, eighteen and thirty weeks) will be collected in order to provide some preliminary data on outcomes such as physical activity, motor and non-motor symptoms, depression and anxiety, fatigue, function, self-efficacy and quality of life. This will generate data on effect sizes to inform sample size calculation for a future effectiveness study. A suite of outcome measures will be employed in order to inform selection of the most appropriate measures for use in a future effectiveness study. All measures will be conducted by the principal researcher who is blind to the intervention allocation, with all measures conducted within the home environment. In the event that Covid-19 restrictions do not permit face-to-face visits, measurements will undertaken using Microsoft Teams, with participants being sent self-administered questionnaires in the post. This will require two measures to be omitted (Mini-BESTest, and item 29 of the UPDRS) to maintain participant safety.

Motor function will be measured by: Unified Parkinson's Disease Rating Scale (UPDRS), Timed up and Go, Functional Gait Assessment, MiniBESTest, six-minute walk test, 10-metre walk test, Schwab and England, and Activities-specific Balance Confidence Scale. All measures are recommended by the Movement Disorders Task Force ${ }^{49-51}$ and have recognised psychometric properties for PwP. In addition, physical activity will be measured by the use of wrist-worn activity tracker, with self-rated activity reported within an activity diary.

Non-motor function will be measured by: UPDRS, Lille Apathy scale, Parkinson's Anxiety scale, Parkinson's Fatigue Scale, Geriatric depression scale, Parkinson's disease questionnaire 39, Nottingham Health Profile. All these measures are recommended by the Movement Disorders Task Force ${ }^{52-56}$ and have recognised psychometric properties for People with Parkinson's. In addition, the self-efficacy scale for exercise, which has been used widely in long-term conditions, and the Warwick Edinburgh Mental Health and Wellbeing Scale, which has established reliability for use in the general population ${ }^{57}$, will be used. The total estimated to complete all measures is 90 minutes.

\section{Planned data analysis}

As this is an acceptability and feasibility study, the analysis will focus on the key parameters necessary for conducting a future trial. Most of the analysis will be descriptive in nature. The principal researcher will intelligently transcribe qualitative data from the semi-structured interviews. Participants will be sent completed transcribed documents to check for accuracy and make amendments or additions as required. Data will be analysed using NVivo version 12; following a framework analysis approach ${ }^{58}$ Coding and categorisation of data will be reviewed by a second researcher. Quantitative data including feasibility measures, demographic information, and baseline measures will be analysed using simple descriptive statistics using SPSS. Preliminary data will also be analysed to estimate the size of effect, by analysing the difference of mean change between PD Connect and Standard care at set points of six, 18 and 30 weeks. This will be calculated for each quantitative outcome measure (e.g. step count as measured by the fitness tracker, strength, balance, UPDRS, QoL) and will be established at each time point in order to calculate effect sizes and inform future sample size calculation. Further, this will inform the primary outcome for use in the future effectiveness study.

\section{Monitoring}

Analysis will be undertaken at set points during the study, following baseline measurements, and after the six, 18 and 30 weeks measurements are conducted. In a review of Parkinson's clinical trials, Allen et al. (2012) reported that $69 \%$ of interventions retained $85 \%$ or more of their participants. Owing to the small sample size in the current feasibility study $(n=30)$, an $85 \%$ retention rate has been set within the PDConnect study, with retention rate below this figure deemed as criterion for not progressing to a full trial. The target adherence for this study is set at $77 \%$ allowing participants to miss one physiotherapy session, and a maximum of three out of 12 weeks of group-based exercise. With exercise participation there is a potential falls risk. Within the activity dairy participants randomised to either arm of the study are asked to note all falls within their activity diary and contact the researcher directly to report any falls. A priori, more than five people reporting a falls would trigger referral to the Data Management Committee (DMC) to discussion potential for study cessation. The DMC for this study aligns with the National Institute for Health Research Guidelines. The DMC will consist of four people who are independent to the study and institution where this study is being hosted. This will include a statistician, a Parkinson's specialist clinician, a senior researcher, and a person with Parkinson's. Meetings will be held annually, or more frequently if required, with a minimum quoracy for all meetings to be $67 \%$ (two thirds) of appointed members. The study will be monitored by all members of the project management group. They will oversee the conduct of the trial. As the sponsor, Robert Gordon University may request to audit processes and procedure, as will NHS Grampian who provide Research and Development (R\&D) site approval for this study.

\section{Ethics and dissemination}

The study was approved by the Liverpool Central REC Centre (IRAS Number 280159) and has local NHS Grampian Research 
and Development approval (2020RG001E). Any protocol modifications required will be communicated by the principal researcher to the ethics and $\mathrm{R} \& \mathrm{D}$ departments, and updated on ISRCTN and reported in publications arising from the study. All participants will be asked to provide signed informed consent (Extended data $^{45}$ ) to participate in this study. Consent will be taken by the principal researcher in the participant's home. Participation in this study will not negate referral to ancillary services such as occupational therapy, should an identified need arise. Any need for compensation will be addressed through the sponsors insurance. All data will be stored in line with GDPR guidelines. Project data, and materials sent for publication, will be anonymised by removing statements identifying participants. The data will be stored securely in a password-protected research drive within the research teams' institution accessible only to the research team. Only the research team will have access to the final data set, and acknowledging this is a feasibility study, data will not be publicly available after completion of the study. The anonymised findings will be included in the first author's doctoral thesis as well as being disseminated through peer-reviewed academic journals, national and international conferences and public events.

\section{Discussion}

Exercise has been shown to slow down the rate of progression of Parkinson's. However, a significant proportion of PwP remain inactive. Current exercise programmes are delivered short term, in isolation and fail to provide PwP the strategies and skills to support long term sustained behaviour change. PDConnect is an innovative intervention combining self-management, and exercise, fostering a collaborative approach between physiotherapy, fitness instructors and PwP. This combination may promote long-term exercise adherence amongst $\mathrm{PwP}$ by providing them with the required skills, knowledge and abilities to participate in exercise. This feasibility and acceptability study will determine recruitment and retention rates, testing the procedures and exploring the acceptability of the programme as well as investigating potential changes in motor, non-motor and health and wellbeing outcomes. This study is in line with the MRC guidance for developing complex interventions (testing procedures, estimating recruitment and retention, determining sample size). This study will not be able to determine effectiveness, but will inform whether PDConnect is feasible to deliver and acceptable to PwP. The quantitative data will provide an initial understanding of the potential benefits of
PDConnect, with qualitative data serving to increase understanding of the perceptions of those participating within PDConnect. The results will indicate the feasibility and acceptability of the intervention, which will inform modifications to the intervention, processes and procedures prior to embarking on a larger scale study, in order to investigate the full effectiveness and cost-effectiveness of the programme.

\section{Trial information}

This study was registered on ISRCTN (ISRCTN11672329, $4^{\text {th }}$ June 2020).

Trial sponsor is Vice-Principal for Research and Commercialisation, c/o Jill Johnston, Robert Gordon University, Aberdeen (j.johnston4@rgu.ac.uk).

\section{Study status}

This study has been awarded full Ethical and Research and Development Approval, and has commenced recruitment.

\section{Data availability}

Underlying data

No underlying data are associated with this article.

\section{Extended data}

Open Science Framework: A collaborative approach to exercise provision for people with Parkinson's - a feasibility and acceptability study of the PDConnect programme, https://doi.org/10.17605/OSF.IO/VWGNT ${ }^{45}$.

The project contains the following extended data:

- Interview topic guide

- Participant information sheet

- Consent form

- Study protocol

\section{Reporting guidelines}

SPIRIT checklist for "A collaborative approach to exercise provision for people with Parkinson's - a feasibility and acceptability study of the PDConnect programme", https://doi.org/ 10.17605/OSF.IO/VWGNT ${ }^{45}$.

Data are available under the terms of the Creative Commons Attribution 4.0 International license (CC-BY 4.0).
1. Pringsheim T, Jette N, Frolkis A, et al.: The prevalence of Parkinson's disease: a systematic review and meta-analysis. Mov Disord. 2014; 29(13): 1583-1590. PubMed Abstract | Publisher Full Text

2. GBD 2016 Parkinson's Disease Collaborators: Global, regional, and national burden of Parkinson's disease, 1990-2016: a systematic analysis for the Global Burden of Disease Study 2016. Lancet Neurol. 2018; 17(11): 939-953. PubMed Abstract | Publisher Full Text | Free Full Text

3. Hetchner MC, Vogt T, Zollner Y, et al:: Quality of life in Parkinson's disease patients with motor fluctuations and dyskinesias in five European countries. Parkinsonism Relat Disord. 2014; 20(9): 969-74. PubMed Abstract | Publisher Full Text
4. American College of Sports Medicine: ACSM's guidelines for exercise testing and prescription. Philadelphia: Lippincott Williams \& Wilkins. 2000. Reference Source

5. Klingelhoefer $\mathrm{L}$, Reichmann $\mathrm{H}$ : Parkinson's disease as a multisystem disorder. J Neural Transm (Vienna). 2017; 124(6): 709-713.

PubMed Abstract | Publisher Full Text

6. Cusso ME, Donald KJ, Khoo TK: The Impact of Physical Activity on NonMotor Symptoms in Parkinson's Disease: A Systematic Review. Front Med (Lausanne). 2016; 3: 35

PubMed Abstract | Publisher Full Text | Free Full Text

7. Blake $H, M o P$, Malik $S$, et al.: How effective are physical activity 
interventions for alleviating depressive symptoms in older people? A systematic review. Clin Rehabil. 2009; 23(10): 873-887. PubMed Abstract | Publisher Full Text

8. Appleman E, Stavitsky K, Cronin-Golomb A: Relation of subjective quality of life to motor symptom profile in Parkinson's disease. Parkinsons Dis. 2011; 2011: 472830

PubMed Abstract | Publisher Full Text | Free Full Text

9. Van Uem JMT, Marinus J, Canning C, et al.: Health-Related Quality of Life in patients with Parkinson's disease--A systematic review based on the IC model. Neurosci Biobehav Rev. 2016; 61: 26-34. PubMed Abstract | Publisher Full Text

10. Lord S, Godfrey A, Galna B, et al.: Ambulatory activity in incident Parkinson's: more than meets the eye? J Neurol. 2013; 260(12): 2964-72. PubMed Abstract | Publisher Full Text

11. Mantri S, Wood S, Duda J, et al.: Comparing self-reported and objective monitoring of physical activity in Parkinson disease. Parkinsonism Relat Disord. 2019; 67: 56-59. PubMed Abstract | Publisher Full Text

12. Shalesh A, Roushdy T, Essam M, et al.: Mental Health, Physical Activity, and Quality of Life in Parkinson's Disease During COVID-19 Pandemic. Mov Disord. 2020; 35(7): 1097-1099.

PubMed Abstract | Publisher Full Text | Free Full Text

13. Simpson J, Eccles F, Doyle C: The impact of coronavirus restrictions on people affected by Parkinson's: the findings from a survey by Parkinson's UK. 2020. Accessed Oct 2020 Reference Source

14. Paillard T, Rolland Y, de Souto Barreto B: Protective Effects of Physical Exercise in Alzheimer's Disease and Parkinson's Disease: A Narrative Review. J Clin Neurol. 2015; 11(3): 212-9.

PubMed Abstract | Publisher Full Text | Free Full Text

15. Klamroth S, Steib S, Devan S, et al.: Effects of Exercise Therapy on Postural Instability in Parkinson Disease: A Meta-analysis. J Neurol Phys Ther. 2016; 40(1): $3-14$

PubMed Abstract | Publisher Full Text

16. Hirsch MA, Iyer S, Sanjak M: Exercise-induced neuroplasticity in human Parkinson's disease: What is the evidence telling us? Parkinsonism Relat Disord. 2016; 22 Suppl 1: 578-eS81. PubMed Abstract | Publisher Full Text

17. Hirsch MA, van Wegen EEH, Newman MA, et al:: Exercise-induced increase in brain-derived neurotrophic factor in human Parkinson's disease: a systematic review and meta-analysis. Trans/ Neurodegener. 2018; 7: 7. PubMed Abstract | Publisher Full Text | Free Full Text

18. Lima LO, Scianni A, Rodrigues-de-Paula F: Progressive resistance exercise improves strength and physical performance in people with mild to moderate Parkinson's disease: a systematic review. J Physiother. 2013; 59(1): $7-13$.

PubMed Abstract | Publisher Full Text

19. Lauze M, Daneault JF, Duval C: The Effects of Physical Activity in Parkinson's Disease: A Review. J Parkinsons Dis. 2016; 6(4): 685-698. PubMed Abstract | Publisher Full Text | Free Full Text

20. Petzinger GM, Fisher BE, McEwen S, et al.: Exercise-enhanced neuroplasticity targeting motor and cognitive circuitry in Parkinson's disease. Lancet Neurology. 2013; 12(7): 716-726.

PubMed Abstract | Publisher Full Text | Free Full Text

21. Frazzitta G, Maestri R, Bertotti G, et al.: Intensive rehabilitation treatment in early Parkinson's disease: a randomized pilot study with a 2-year followup. Neurorehabil Neural Repair. 2015; 29(2): 123-131.

PubMed Abstract | Publisher Full Text

22. Ahsklog JE: Does vigorous exercise have a neuroprotective effect in Parkinson disease? Neurology. 2011; 77(3): 288-294. PubMed Abstract | Publisher Full Text | Free Full Text

23. Ellis T, Rochester L: Mobilizing Parkinson's Disease: The Future of Exercise. J Parkinsons Dis. 2018; 8(s1): S95-S100.

PubMed Abstract | Publisher Full Text | Free Full Text

24. Chief Medical Office: Physical Activity Guidelines. [accessed June 2020] Reference Source

25. Parkinson's UK: Exercise framework. 2018. [accessed June 2020] Reference Source

26. Hulbert SM, Goodwin VA: 'Mind the gap' - a scoping review of long term, physical, self-management in Parkinson's. Physiotherapy. 2020; 107: 88-99. PubMed Abstract | Publisher Full Text

27. Allen N, Sherrington $C$, Suriyarachchi GD, et al:: Exercise and motor training in people with Parkinson's disease: a systematic review of participant characteristics, intervention delivery, retention rates, adherence, and adverse events in clinical trials. Parkinsons Dis. 2012; 2012: 854328. adverse events in clinical trials. Parkinsons Dis. 2012; 2012
PubMed Abstract | Publisher Full Text | Free Full Text

28. Clarke CE, Patel $\mathrm{S}$, Ives $\mathrm{N}$, et al: : Physiotherapy and Occupational Therapy vs No Therapy in Mild to Moderate Parkinson Disease: A Randomized Clinical Trial. JAMA Neurol. 2016; 73(3): 291-299. PubMed Abstract | Publisher Full Text

29. Collect J, Franssen M, Meaney A, et al.: Phase II randomised controlled trial of a 6-month self-managed community exercise programme for people with
Parkinson's disease.J Neurol Neurosurg Psychiatry. 2017; 88(3): 204-211. PubMed Abstract | Publisher Full Text

30. Wagner $\mathrm{EH}$ : Chronic disease management: what will it take to improve care for chronic illness? Eff Clin Pract. 1998; 1(1): 2-4 PubMed Abstract

31. Vlaanderen F, Rompen L, Munneke M, et al:: The Voice of the Parkinson Customer.J Parkinsons Dis. 2019; 9(1): 197-201. PubMed Abstract | Publisher Full Text

32. Scottish Government: Scotlands digital Health and social care strategy: enabling, connecting and empowering. 2019; accessed Oct 2020. Reference Source

33. Ellis T, Boudreau J, DeAngelis T, et al:: Barriers to exercise in people with Parkinson disease. Phys Ther. 2013; 93(5): 628-36. PubMed Abstract | Publisher Full Text | Free Full Text

34. Pappa S, Brundin P, Fung V, et al:: Impact of the COVID-19 Pandemic on Parkinson's Disease and Movement Disorders. Mov Disord. 2020; 35(5): 711-715

PubMed Abstract | Publisher Full Text

35. Thomas S, Mancini F, Ebenezer L, et al.: Parkinson's disease and the COVID-19 pandemic: responding to patient need with nurse-led telemedicine. British Journal of Neuroscience Nursing. 2020; 16(3): 131-133. Publisher Full Text

36. Schirinzi T, Cerroni R, Di Lazzaro G, et al.: Self-reported needs of patients with Parkinson's disease during COVID-19 emergency in Italy. Neurol Sci. 2020; 41(6): 1373-1375

PubMed Abstract | Publisher Full Text | Free Full Text

37. Wilkinson JR, Spindler M, Wood SM, et al.: High patient satisfaction with telehealth in Parkinson disease: A randomized controlled study. Neurol Clin Pract. 2016; 6(3): 241-251.

PubMed Abstract | Publisher Full Text | Free Full Text

38. Beck C, Beran D, Biglan $\mathrm{K}$, et al.: National randomized controlled trial of virtual house calls for Parkinson disease. Neurology. 2017; 89(11): 1152-1161. PubMed Abstract | Publisher Full Text | Free Full Text

39. Flynn A, Allen N, Dennis $S$, et al:: Home-based prescribed exercise improves balance-related activities in people with Parkinson's disease and has benefits similar to centre-based exercise: a systematic review. J Physiother. 2019: 65(4): 189-199.

PubMed Abstract | Publisher Full Text

40. Van der Kolk N, de Vries N, Kessles R, et al.: Effectiveness of home-based and remotely supervised aerobic exercise in Parkinson's disease: a doubleblind, randomised controlled trial. Lancet Neurol. 2019; 18(11): 998-1008. PubMed Abstract | Publisher Full Text

41. Landers M, Ellis T: A Mobile App Specifically Designed to Facilitate Exercise in Parkinson Disease: Single-Cohort Pilot Study on Feasibility, Safety, and Signal of Efficacy. JMIR Mhealth Uhealth. 2020; 8(10): e18985. PubMed Abstract | Publisher Full Text | Free Full Text

42. Craig, et al.: Medical Research Council: Developing and evaluating complex interventions. 2008; [accessed June 2020]. Reference Source

43. Conradsson $\mathrm{D}$, Nero $\mathrm{H}$, Lofgren $\mathrm{H}$, et al:: Monitoring training activity during gait-related balance exercise in individuals with Parkinson's disease: a proof-of-concept-study. BMC Neurol. 2017; 17(1): 19. PubMed Abstract | Publisher Full Text | Free Full Text

44. Harvey M, Weston $\mathrm{K}$, Gray W, et al.: High-intensity interval training in people with Parkinson's disease: a randomized, controlled feasibility trial. Clin Rehabil. 2019; 33(3): 428-438. PubMed Abstract | Publisher Full Text

45. Jones J, Cooper K, Professor H, et al.: A collaborative approach to exercise provision for people with Parkinson's - a feasibility and acceptability study of the PDConnect programme. 2020. http://www.doi.org/10.17605/OSF.IO/VWGN

46. Keus S, Munnke M, Graziano M, et al.: European Physiotherapy Guideline for Parkinson's Disease. KNGP/ParkinsonsNet, The Netherlands. 2014. Reference Source

47. Borg G: Psychophysical bases of perceived exertion. Med Sci Sports Exerc. 1982; 14(5): 377-381. PubMed Abstract

48. Creswell J, Plano-Clark V: Designing and conducting mixed methods research. $2^{\text {nd }}$ Edition. Sage publishing: London. 2011. Reference Source

49. Goetz C, Tiley B, Shaftman S, et al.: Movement Disorder Society-sponsored revision of the Unified Parkinson's Disease Rating Scale (MDS-UPDRS): scale presentation and clinimetric testing results. Mov Disord. 2008; 23(15): 2129-2170.

PubMed Abstract | Publisher Full Text

50. Bloem B, Marinus J, Almeida Q, et al.: Measurement instruments to assess posture, gait, and balance in Parkinson's disease: Critique and recommendations. Mov Disord. 2016; 31(9): 1342-1355. PubMed Abstract | Publisher Full Text

51. Shulman $L$, Armstrong $M$, Ellis $T$, et al.: Disability Rating Scales in Parkinson's Disease: Critique and Recommendations. Mov Disord. 2016; 31(10): 1455-1468. PubMed Abstract | Publisher Full Text 
52. Friedman J, Alves G, Hagell P, et al.: Fatigue rating scales critique and recommendations by the Movement Disorders Society task force on rating scales for Parkinson's disease. Mov Disord. 2010; 25(7): 805-822. PubMed Abstract | Publisher Full Text

53. Leentjens A, Dujardin K, Marsh L, et al:: Apathy and anhedonia rating scales in Parkinson's disease: critique and recommendations. Mov Disord. 2008a; 23(14): 2004-2014. PubMed Abstract | Publisher Full Text

54. Leentjens AFG, Dujardin K, Marsh L, et al.: Anxiety rating scales in Parkinson's disease: critique and recommendations. Mov Disord. 2008b; 23(14): 2015-2025.

PubMed Abstract | Publisher Full Text

55. Schrag A, Barone P, Brown RG, et al.: Depression rating scales in Parkinson's disease: critique and recommendations. Mov Disord. 2007; 22(8): 1077-1092. PubMed Abstract | Publisher Full Text | Free Full Text

56. Martinez-Martin P, Jeukens-Visser M, Lyons K, et al.: Health-related qualityof-life scales in Parkinson's disease: critique and recommendations. Mov Disord. 2011; 26(13): 2371-2380.

PubMed Abstract | Publisher Full Text

57. Tennant $\mathrm{R}$, Hiller $\mathrm{L}$, Fishwick $\mathrm{R}$, et al.: The Warwick-Edinburgh Mental Wellbeing Scale (WEMWBS): development and UK validation. Health Qual Life Outcomes. 2007; 5: 63.

PubMed Abstract | Publisher Full Text | Free Full Text

58. Ritchie J, Lewis J: Qualitative research practice: a guide for social science students and researchers. London: Sage. 2003. Reference Source 


\title{
Open Peer Review
}

\section{Current Peer Review Status: ? ?}

\section{Version 1}

Reviewer Report 29 January 2021

https://doi.org/10.21956/amrcopenres.14007.r26601

(c) 2021 Morris R. This is an open access peer review report distributed under the terms of the Creative Commons Attribution License, which permits unrestricted use, distribution, and reproduction in any medium, provided the original work is properly cited.

\section{Rosie Morris}

Department of Sport, Exercise, and Rehabilitation, Northumbria University, Newcastle upon Tyne, UK

The study by Jones et al aims to establish whether their intervention study, PDConnect, is feasible and acceptable in a cohort of patients with Parkinson's disease. The aim of this intervention is to promote both physical activity and self-management for exercise for these patients. This is an interesting study and timely given the current pandemic. The protocol is clear and well written, there are a number of minor points that could be addressed before indexing.

\begin{abstract}
The abstract is clear and concise. The word 'limiting' in 'limiting the rate of decline...' may want to be changed to 'slowing', but this is a minor point.
\end{abstract}

\section{Introduction}

The introduction of the manuscript is comprehensive and provides a good background to the study. There are a few minor points the authors could improve on:

Throughout the introduction there is discussion around Covid-19 and the influence this has had on face-to-face interventions. Covid-19 has provided a catalyst for seeking virtual interventions; however, the importance of virtual interventions should be recognised further than Covid-19. For example, the need for this treatment for those who live remotely, reduced costs, reduced time required, increased retention rates etc.

Figure 1 is a little confusing. At present it suggests that impact of diagnosis influences NMS and NMS influence motor symptoms. Perhaps this figure could be altered to represent that Protocol the four factors separately impact negatively on physical activity participation.

In the inclusion criteria, does confirmed diagnosis of PD include meeting UK Brain Bank Criteria?

Within the criteria, could the authors also include H\&Y IV, is there a rationale for removing 
these participants?

Spelling error within Hoehn and Yahr under 'Randomisation and Binding'

Within the project protocol it is highlighted that specialist Physiotherapists with training will be involved in the PDConnect group but not the usual care group. As the main outcome of the study is addressing self-management, does this require those specialist skills? Perhaps the difference in training may influence the outcomes of the study

The protocol states that exercise needs to be completed five times per week. Within the introduction there is a suggestion that daily exercise is required. It is appreciated that it would be difficult to retain participants if daily exercise was expected. But does this need to be addressed within the self-management programme?

Within the outcome measures, please state which sections of the UPDRS will be utilised for both motor and non-motor symptoms and whether you are using the MDS-UPDRS or the UPDRS

It may be worth including cognitive assessments within the non-motor symptoms outcome measures due to the benefit of exercise on cognition

An activity tracker is going to be used to monitor physical activity. Which monitor is going to be used and is there a reference for validity and reliability?

Is the rationale for, and objectives of, the study clearly described?

Yes

Is the study design appropriate for the research question?

Yes

Are sufficient details of the methods provided to allow replication by others? Partly

Are the datasets clearly presented in a useable and accessible format? Yes

Competing Interests: No competing interests were disclosed.

Reviewer Expertise: Ageing, Neurodegenerative disease, Physiotherapy, mobility (gait, balance, falls), cognition

I confirm that I have read this submission and believe that I have an appropriate level of expertise to confirm that it is of an acceptable scientific standard, however I have significant reservations, as outlined above.

Reviewer Report 13 January 2021 


\section{https://doi.org/10.21956/amrcopenres.14007.r26584}

(C) 2021 Allen N. This is an open access peer review report distributed under the terms of the Creative Commons Attribution License, which permits unrestricted use, distribution, and reproduction in any medium, provided the original work is properly cited.

\section{Natalie Allen}

Discipline of Physiotherapy, Faculty of Medicine and Health, University of Sydney, Sydney, Australia

This protocol is for a randomised, mixed methods study exploring the acceptability and feasibility of a multicomponent intervention aimed at increasing physical activity and improving exercise self-management in people with mild to moderate Parkinson's disease (PD). The benefits of exercise for people with PD are well established, yet most people with PD do not perform the recommended amount. Therefore, this project addresses the important issue of how to establish and support long term exercise participation amongst people with PD. The project intervention is delivered via telehealth, and therefore complies with limitations due to the Covid-19 pandemic, as well as providing important information about the acceptability and feasibility of telehealth that can be used in the future (e.g., for people living in rural and remote areas, or who are unable to travel). The project is designed to provide the information required to design an appropriately powered randomised controlled trial exploring the effectiveness of the intervention.

General comments

The concept of self-management is clearly explained and justified; however, the self-management component of the program appears to be about exercise self-management (rather than selfmanagement more broadly). I suggest this is directly clarified in the text (e.g., by using the term 'exercise self-management' where applicable.

Some abbreviations did not appear to be written in full on the first use - eg PWP, MRC

\section{Abstract}

The abstract is written clearly and contains the key information.

\section{Introduction}

The introduction sets up the rationale for the project and is written in a clear and logical sequence. However, there are two sentences near the end of the second last paragraph that are written poorly and should be revised for clarity and English expression (starting from "The use of a video guided exercise health app...")

Please consider revising Figure 1. What do the arrows represent? To me, the arrows suggest that one thing is leading to or causing the other, but in this figure this is not necessarily the case. For example, non-motor symptoms do not lead to or cause motor symptoms. The figure legend and associated information in the text suggest the figure shows how these factors influence physical activity, however this is not clear in the figure itself.

\section{Protocol}

This is a complex intervention, and therefore I expect it is difficult to clearly describe. The research protocol in the extended data is very helpful - I suggest referring to this document in the main text, as has been done for the topic guide, consent form etc. While the overall design is clear, and a research protocol is provided in the extended data, there are some details that I think should be 
clarified in the main text.

Please describe how the BCTs will be embedded into the specialist physiotherapy intervention. The BCTs are not referenced and it appears that some are from the Behaviour Change Taxonomy while others do not follow taxonomy wording (e.g., developing selfawareness). I suggest using wording from the Behaviour Change Taxonomy and referring to the taxonomy.

Will exercise be progressed during the program? If so, how?

How will the group exercise sessions work? How many people will be in any one group? Will participants be able to see each other and/or the instructor? If so, this could be distracting and raise safety concerns - how will this be managed? How will the exercise level be determined? Will participants be told their level and exercises so they can review the resources on teams prior to the class? Will participants all be doing the same exercise at the same time?

Will there be communication regarding specific participants between the physiotherapist and fitness instructor? How will this be organised? Will the home exercise program used in the 12 weeks of exercise self-management be the same as that developed during the specialised physiotherapy?

Figure 2 - consider adding further information to clarify the location/method of delivery. For example, indicate that consent and assessments will occur in participant's homes via an in-person visit, and that the physiotherapy, fitness instructor exercise group and supported exercise selfmanagement will be using videoconferencing.

Randomisation and blinding: the word 'Yahr' has a typo The level of expertise of the physiotherapists is described in a way that is specific to the UK - I suggest revising this to describe it in a way that an international audience will understand. The volume of intervention in the usual care group is less than the volume of intervention in the PDConnect group. This is likely to influence the secondary outcome measures and should be taken into account when using the secondary outcome results to design and power a large-scale RCT.

The PDConnect program is underpinned by empowerment theory - please provide a reference for empowerment theory.

The information about the time required for the 12 -week group exercise class is inconsistent. The class is to be a minimum of 60 minutes; however, it includes 40 minutes of exercise and 30 minutes of discussion.

Outcome measurement

Acceptability - The sentence stating that "Interviews involving those delivering the intervention will be conducted using Microsoft Teams" appears to be superfluous.

Will field notes or journaling be used alongside the semi-structured interviews?

What will the fidelity assessment be looking for specifically?

Motor function and non-motor function are to be measured using the UPDRS. Are you using the old UPDRS, or the newer, MDS-UPDRS version? A reference would also help to clarify this.

In the motor function measurement section Please specify which activity tracker you will be using, and what the tracker will be measuring (i.e., steps). Is there a justification for wearing the tracker 
on the wrist when this is likely to overestimate physical activity?

Monitoring: the authors note that if more than 5 people report falls, then the data management committee will discuss possible study cessation. Is this any fall, or a fall while undertaking the prescribed exercises? Please clarify. People with PD are known to be at a high risk of falls; therefore, I consider it likely that more than 5 out of 30 people will fall during the course of the study - though hopefully not while undertaking the intervention.

Who are the project management group?

Is the rationale for, and objectives of, the study clearly described?

Yes

Is the study design appropriate for the research question?

Yes

Are sufficient details of the methods provided to allow replication by others?

No

Are the datasets clearly presented in a useable and accessible format?

Yes

Competing Interests: No competing interests were disclosed.

Reviewer Expertise: My research focuses on exercise and physiotherapy-based interventions for people with Parkinson's disease.

I confirm that I have read this submission and believe that I have an appropriate level of expertise to confirm that it is of an acceptable scientific standard, however I have significant reservations, as outlined above.

\section{Comments on this article}

\section{Version 1}

Author Response 19 Mar 2021

Julie Jones, Robert Gordon University, Aberdeen, UK

"Thank you for your comments and feedback in relation to this study protocol. The feedback to each comment is written in italics below to that the responses can be easily distinguished from the reviewer's comments".

\section{Rosie Mearns comments}

Abstract

The abstract is clear and concise. The word 'limiting' in 'limiting the rate of decline...' may want to be 
changed to 'slowing', but this is a minor point.

"Thank you for this suggestion, we have changed the wording, encompassing slowing"

\section{Introduction}

The introduction of the manuscript is comprehensive and provides a good background to the study. There are a few minor points the authors could improve on:

Throughout the introduction there is discussion around Covid-19 and the influence this has had on face-to-face interventions. Covid-19 has provided a catalyst for seeking virtual interventions; however, the importance of virtual interventions should be recognised further than Covid-19. For example, the need for this treatment for those who live remotely, reduced costs, reduced time required, increased retention rates etc.

"The introduction text has been amended to reflect that Telehealth was gaining momentum prior to the Covid-19 pandemic and has seen rapid deployment across many healthcare sectors, including exercise delivery".

Figure 1 is a little confusing. At present it suggests that impact of diagnosis influences NMS and NMS influence motor symptoms. Perhaps this figure could be altered to represent that the four factors separately impact negatively on physical activity participation

"A new figure 1 has been submitted in version 2 of the article"

\section{Protocol}

In the inclusion criteria, does confirmed diagnosis of PD include meeting UK Brain Bank Criteria? "We have not used the UK Brain Bank Criteria - instead, diagnosis is made by Parkinson's specialist reflecting local clinical practice. This has been clarified within the inclusion criteria"

Within the criteria, could the authors also include H\&Y IV, is there a rationale for removing these participants?

"Rationale for removing stage IV, was to ensure safety with exercising remotely at home. At level four they may or may not be able to stand unassisted, and for safety reasons they were excluded."

Spelling error within Hoehn and Yahr under 'Randomisation and Binding'

"Thank you for highlighting this is now corrected"

Within the project protocol it is highlighted that specialist Physiotherapists with training will be involved in the PDConnect group but not the usual care group. As the main outcome of the study is addressing self-management, does this require those specialist skills? Perhaps the difference in training may influence the outcomes of the study "The provision of training to only those delivering PDConnect is a recognised potential confounder but that the study is comparing PDConnect (a novel combination of specialist physio, exercise \& selfmanagement) with usual care. Therefore, training is required for the specialist PT element. AS this is a feasibility and acceptability study, the finding will be used to refine the proposed intervention theory following this study and include a process evaluation in the RCT to explore assumptions associated with this complex intervention".

The protocol states that exercise needs to be completed five times per week. Within the introduction there is a suggestion that daily exercise is required. It is appreciated that it would be 
difficult to retain participants if daily exercise was expected. But does this need to be addressed within the self-management programme?

"Thank you for highlighting this. Participants will be encouraged to develop exercise habit will be addressed as part of the self-management, and use of BCTs. This will include structured exercise five times week, with promotion of a physically active lifestyle in addition to this. A minor amendment to the introduction have been made to enhance clarity of this".

Within the outcome measures, please state which sections of the UPDRS will be utilised for both motor and non-motor symptoms and whether you are using the MDS-UPDRS or the UPDRS "Guidance was taken from local consultants, with the UPDRS being used owing to the costs and licencing with MDS UPDRS. This has been clarified within the referencing with the version by Fahn et al."

It may be worth including cognitive assessments within the non-motor symptoms outcome measures due to the benefit of exercise on cognition

"Thank you for the suggestion, we agree that the benefit of exercise on cognition is important and did consider this when selecting outcomes. However, following discussion with the study team and advisors, due to the battery of outcomes included and the inclusion of cognition within the UPDRS, we decided not to include a specific cognitive measure"

An activity tracker is going to be used to monitor physical activity. Which monitor is going to be used and is there a reference for validity and reliability?

"Type of tracker, reliability and validity have been added to the manuscript within the section on motor function measures".

"Thank you for your time taken to review this article and we appreciate your feedback".

\section{NATALIE ALLEN COMMENTS}

"Thank you for your comments and feedback, very much appreciated."

General comments

The concept of self-management is clearly explained and justified; however, the self-management component of the program appears to be about exercise self-management (rather than selfmanagement more broadly). I suggest this is directly clarified in the text (e.g., by using the term 'exercise self-management' where applicable.

"The term exercise self-management has been adopted within the manuscript"

Some abbreviations did not appear to be written in full on the first use - eg PWP, MRC "This has been rectified, thank you".

\section{Abstract}

The abstract is written clearly and contains the key information.

\section{Introduction}

The introduction sets up the rationale for the project and is written in a clear and logical sequence. However, there are two sentences near the end of the second last paragraph that are written 
poorly and should be revised for clarity and English expression (starting from "The use of a video guided exercise health app...")

"This has been amended, thank you".

Please consider revising Figure 1. What do the arrows represent? To me, the arrows suggest that one thing is leading to or causing the other, but in this figure this is not necessarily the case. For example, non-motor symptoms do not lead to or cause motor symptoms. The figure legend and associated information in the text suggest the figure shows how these factors influence physical activity, however this is not clear in the figure itself.

A new figure 1 has been submitted in version 2 of the article"

\section{Protocol}

This is a complex intervention, and therefore I expect it is difficult to clearly describe. The research protocol in the extended data is very helpful - I suggest referring to this document in the main text, as has been done for the topic guide, consent form etc. While the overall design is clear, and a research protocol is provided in the extended data, there are some details that I think should be clarified in the main text.

"The research protocol is now referred to in the main text within the amended submission".

Please describe how the BCTs will be embedded into the specialist physiotherapy intervention. The BCTs are not referenced and it appears that some are from the Behaviour Change Taxonomy while others do not follow taxonomy wording (e.g., developing self-awareness). I suggest using wording from the Behaviour Change Taxonomy and referring to the taxonomy.

"Specific Taxonomy has now been referenced within the manuscript, thank you for highlighting this omission. The wording has been adjusted so it aligns with the terminology used within the behaviour change taxonomy within the manuscript, so it is clearer for the reader"

Will exercise be progressed during the program? If so, how?

"Yes, exercise will be progressed within the programme. This will be done on an individual basis, to ensure that all exercise is progressive in nature following the guidelines outlined in the European Physiotherapy Guidelines for Parkinson's".

Text has been added within the manuscript to clarify this point you have raised. How will the group exercise sessions work? How many people will be in any one group? Will participants be able to see each other and/or the instructor? If so, this could be distracting and raise safety concerns - how will this be managed? How will the exercise level be determined? "There will be a maximum of 8 PWP in a group, aligning with best practice guidelines for safe delivery of online exercise. The manuscript has been edited to reflect this. Participants will be able to see each other on screen using Microsoft teams. However during classes, participants would be advised to have their screen on speaker view, so they can focus on the instructor and not be distracted by other participants. The PI will have conducted an online home risk assessment and completed a participant information statement prior to commencing the study. This has been added to the manuscript."

Will participants be told their level and exercises so they can review the resources on teams prior to the class? Will participants all be doing the same exercise at the same time? "Yes, participants will be told their levels and videos of the exercises will be available on the Microsoft 
teams site prior to the class. All participants will all be doing the same exercise as the same time, however the level of that exercise may differ depending on individual ability. These points have been clarified with the amended version of the manuscript."

Will there be communication regarding specific participants between the physiotherapist and fitness instructor? How will this be organised? Will the home exercise program used in the 12 weeks of exercise self-management be the same as that developed during the specialised physiotherapy?

"Thank you for highlighting this. A summary document will be used including a summary of PT, HEP, participants current goals. This will be saved on a closed channel (ie so participants cannot access this) within the PDConnect Microsoft Teams area. The manuscript has been edited to reflect this. The HEP developed in the 6 weeks of PT will be continued and developed during the 12 weeks group based element of the programme."

Figure 2 - consider adding further information to clarify the location/method of delivery. For example, indicate that consent and assessments will occur in participant's homes via an in-person visit, and that the physiotherapy, fitness instructor exercise group and supported exercise selfmanagement will be using videoconferencing.

"Figure 2 has been updates to reflect your comments"

Randomisation and blinding: the word 'Yahr' has a typo

"This has been corrected, thank you."

The level of expertise of the physiotherapists is described in a way that is specific to the UK - I suggest revising this to describe it in a way that an international audience will understand. "Thank you, this has been amended, to two years post qualify experience."

The volume of intervention in the usual care group is less than the volume of intervention in the PDConnect group. This is likely to influence the secondary outcome measures and should be taken into account when using the secondary outcome results to design and power a large-scale RCT. "This factor has been mentioned within the discussion section of the discussion section. Similarly with the above point made in relation to specialist training of the physiotherapist, this will be further considered when designing the future RCT."

The PDConnect program is underpinned by empowerment theory - please provide a reference for empowerment theory.

"Added within manuscript"

The information about the time required for the 12-week group exercise class is inconsistent. The class is to be a minimum of 60 minutes; however, it includes 40 minutes of exercise and 30 minutes of discussion.

"Thank you for highlighting this inconsistency. Participants will engage in 10 stations of exercise each lasting 4 mins, and there will be an additional 10 minutes for warm and cool down, prior to the 30 minute discussion. This will make up the 90 Minute session. This has been clarified within the manuscript."

Outcome measurement 
Acceptability - The sentence stating that "Interviews involving those delivering the intervention will be conducted using Microsoft Teams" appears to be superfluous.

"The word has been removed, thank you."

Will field notes or journaling be used alongside the semi-structured interviews?

"Yes, journal notes will also be used alongside the semi structured interviews, this has been added to the manuscript."

What will the fidelity assessment be looking for specifically?

"Fidelity assessment is to ascertain that the intervention is being delivered as planned, in particular the duration of time spent exercising, content and the style of delivery. Further clarification on this has been added to the manuscript"

Motor function and non-motor function are to be measured using the UPDRS. Are you using the old UPDRS, or the newer, MDS-UPDRS version? A reference would also help to clarify this.

"Reference has been added to the manuscript to clarify this point, thank you."

In the motor function measurement section Please specify which activity tracker you will be using, and what the tracker will be measuring (i.e., steps). Is there a justification for wearing the tracker on the wrist when this is likely to overestimate physical activity?

"The Mi band tracker will be used. The name of this tracker and it reliability and validity have been added to the transcript. Although wrist worn tracker are known to be less reliable. They were selected over LL or body worn devices due to participant convenience"

Monitoring: the authors note that if more than 5 people report falls, then the data management committee will discuss possible study cessation. Is this any fall, or a fall while undertaking the prescribed exercises? Please clarify. People with PD are known to be at a high risk of falls; therefore, I consider it likely that more than 5 out of 30 people will fall during the course of the study - though hopefully not while undertaking the intervention.

"Only falls during the intervention will be considered, the manuscript has been amended to reflect this".

Many thanks indeed for the time taken to review this article and for all the constructive comments, this has been much appreciated."

Competing Interests: Nil 